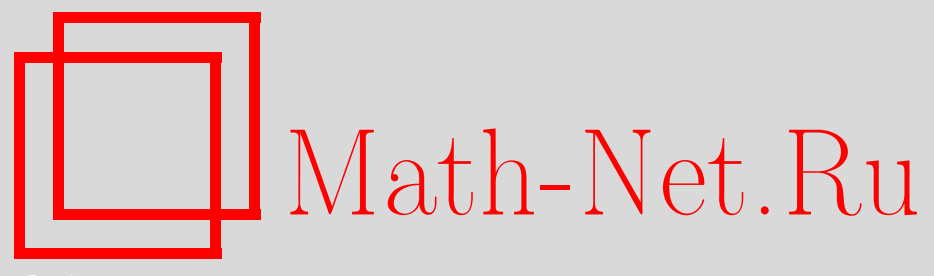

А. Н. Ширяев, Доказательство неравенства ПуанкареЧернова и логарифмического неравенства Соболева методами стохастического исчисления для броуновского движения, УМН, 2006, том 61, выпуск 3, 177-178

DOI: https://doi.org/10.4213/rm1756

Использование Общероссийского математического портала Math-Net.Ru подразумевает, что вы прочитали и согласны с пользовательским соглашением http://www . mathnet.ru/rus/agreement

Параметры загрузки:

IP: 3.82 .47 .9

26 апреля 2023 г., 17:01:03

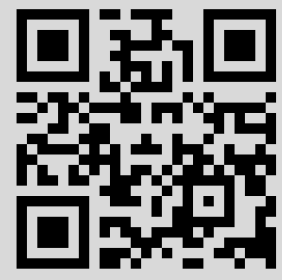




\section{Доказательство неравенства Пуанкаре-Чернова и логарифмического неравенства Соболева методами стохастического исчисления для броуновского движения}

\section{А. Н. Ширяев}

1. Пусть $\xi=\xi(\omega)$ - стандартная нормальная, $\mathscr{N}(0,1)$, случайная величина, функция $g=g(x) \in C^{1}, x \in \mathbb{R}, \mathrm{E} g(\xi)=0$ и $\mathrm{E} g^{2}(\xi)<\infty$. Известное неравенство ПуанкареЧернова (см., например, [1]-[6]) утверждает, что

$$
\mathrm{E} g^{2}(\xi) \leqslant \mathrm{E}\left(g^{\prime}(\xi)\right)^{2} .
$$

(Без предположения $\mathrm{E} g(\xi)=0$ это неравенство имеет вид $\mathrm{D} g(\xi) \leqslant \mathrm{E}\left(g^{\prime}(\xi)\right)^{2}$.)

Если $g \in C^{1}, \mathrm{E} g^{2}(\xi)=1$ и $\mathrm{E}\left(g^{\prime}(\xi)\right)^{2}<\infty$, то оказывается, что $\mathrm{E} g^{2}(\xi) \log g^{2}(\xi)<\infty$ (иными словами, если $g \in L^{2}, g^{\prime} \in L^{2}$, то $g \in L^{2} \log L^{2}$, где $\|g\|_{L_{2}}^{2}=\int_{\mathbb{R}} g^{2}(x) \varphi(x) d x$ и $\left.\varphi(x)=(2 \pi)^{-1 / 2} e^{-x^{2} / 2}\right)$. Вытекает это из логарифмического неравенства Соболева

$$
\mathrm{E} g^{2}(\xi) \log g^{2}(\xi) \leqslant 2 \mathrm{E}\left(g^{\prime}(\xi)\right)^{2},
$$

установленного впервые в работе Л. Гросса [5]. (Если не предполагать, что выполнено условие $\mathrm{E} g^{2}(\xi)=1$, то это неравенство имеет такой вид: энтропия $\mathrm{E} g^{2}(\xi) \log g^{2}(\xi)-$ $\mathrm{E} g^{2}(\xi) \log \mathrm{E} g^{2}(\xi) \leqslant 2 \mathrm{E}\left(g^{\prime}(\xi)\right)^{2} ;$ константа 2 неулучшаема.)

Известны разные доказательства неравенства Пуанкаре-Чернова и логарифмического неравенства Соболева (см., например, [1]-[8]). Ниже мы даем прозрачные доказательства этих неравенств, основанные на идее привлечения броуновского движения и использовании его стандартных свойств.

2. Пусть $B=\left(B_{t}\right)_{t \geqslant 0}$ - стандартное броуновское движение (при $t=1$ распределение $B_{1}$ совпадает с распределением $\xi$, что далее будет использовано). Пусть $\mathrm{E}\left|h\left(B_{T}\right)\right|<\infty$ для некоторого $T>0$, где $h=h(x)$ - измеримая функция, $x \in \mathbb{R}$.

Рассмотрим мартингал $M_{t}=\mathrm{E}\left[h\left(B_{T}\right) \mid \mathscr{F}_{t}\right], t \leqslant T$, где $\mathscr{F}_{t}=\sigma\left(B_{s}, s \leqslant t\right)$. Понятно, что $M_{t}=\mathrm{E}\left[h\left(B_{T}\right) \mid B_{t}\right]=F\left(t, B_{t}\right)$, где $F(t, x)$ - некоторая измеримая функция (в качестве которой можно взять $\left.\mathrm{E}\left[h\left(B_{T}\right) \mid B_{t}=x\right)\right]$ ). Для $F(t, x)$ справедливо обратное уравнение Колмогорова $\frac{\partial F}{\partial t}+\frac{1}{2} \frac{\partial^{2} F}{\partial x^{2}}=0$, и формула Ито дает для каждого $u \leqslant T$, что

$$
F\left(u, B_{u}\right)=F\left(0, B_{0}\right)+\int_{0}^{u} \frac{\partial F}{\partial x}\left(t, B_{t}\right) d B_{t},
$$

T.e.

$$
M_{u}=M_{0}+\int_{0}^{u} \frac{\partial F}{\partial x}\left(t, B_{t}\right) d B_{t}
$$

Если $\mathrm{E}\left|h\left(B_{T}\right)\right|<\infty, \mathrm{E}\left|h^{\prime}\left(B_{T}\right)\right|<\infty$, то интегрированием по частям находим для $F(t, x)=\int_{\mathbb{R}} h(y) \varphi_{T-t}(y \mid x) d y$ с $\varphi_{s}(y \mid x)=(1 / \sqrt{2 \pi s}) e^{-(y-x)^{2} /(2 s)}$, что $\frac{\partial F}{\partial x}(t, x)=$ $\int_{\mathbb{R}} h^{\prime}(y) \varphi_{T-t}(y \mid x) d y=\mathrm{E}\left[h^{\prime}\left(B_{T}\right) \mid B_{t}=x\right]$, и из (4) видим, что для $M_{u}, u \leqslant T$, справедливо стохастическое представление

$$
M_{u}=M_{0}+\int_{0}^{u} \mathrm{E}\left[h^{\prime}\left(B_{T}\right) \mid \mathscr{F}_{t}\right] d B_{t}
$$

и, в частности, для $h\left(B_{T}\right)$ имеет место представление

$$
h\left(B_{T}\right)=\mathrm{E} h\left(B_{T}\right)+\int_{0}^{T} \mathrm{E}\left[h^{\prime}\left(B_{T}\right) \mid \mathscr{F}_{t}\right] d B_{t},
$$

Работа выполнена при поддержке РФФИ (грант № 05-01-00944-а) и Alexander von Humboldt Foundation. 
которое можно было бы получить и из известной формулы Кларка-Окона, требующей обращения к производной Маллявена; см. также [2], [9].

3. Для доказательства неравенства Пуанкаре-Чернова возьмем в (6) $h=g$ с $\mathrm{E} g\left(B_{T}\right)=0, \mathrm{E} g^{2}\left(B_{T}\right)<\infty$ и $\mathrm{E}\left(g^{\prime}\left(B_{T}\right)\right)^{2}<\infty$. По свойствам стохастических интегралов и неравенству Иенсена

$$
\begin{aligned}
\mathrm{E} g^{2}\left(B_{T}\right) & =\mathrm{E} \int_{0}^{T}\left(\mathrm{E}\left[g^{\prime}\left(B_{T}\right) \mid \mathscr{F}_{t}\right]\right)^{2} d t \leqslant \mathrm{E} \int_{0}^{T} \mathrm{E}\left[\left(g^{\prime}\left(B_{T}\right)\right)^{2} \mid \mathscr{F}_{t}\right] d t \\
& =\int_{0}^{T} \mathrm{E}\left(g^{\prime}\left(B_{T}\right)\right)^{2} d t=T \mathrm{E}\left(g^{\prime}\left(B_{T}\right)\right)^{2} .
\end{aligned}
$$

Положив $N_{T}=\mathrm{E}\left[g^{\prime}\left(B_{T}\right) \mid \mathscr{F}_{t}\right], t \leqslant T$, видим, что $N_{t}^{2}, t \leqslant T,-$ субмартингал и, значит, $\mathrm{E} N_{t}^{2} \geqslant \mathrm{E} N_{0}^{2}, t \leqslant T$. Тем самым, из $(7)$ находим: $T\left(\mathrm{E} g^{\prime}\left(B_{T}\right)\right)^{2} \leqslant \mathrm{E} g^{2}\left(B_{T}\right) \leqslant$ $T \mathrm{E}\left(g^{\prime}\left(B_{T}\right)\right)^{2}$, что при $T=1$ дает двустороннее неравенство Пуанкаре-Чернова

$$
\left(\mathrm{E} g^{\prime}(\xi)\right)^{2} \leqslant \mathrm{E} g^{2}(\xi) \leqslant \mathrm{E}\left(g^{\prime}\left(B_{T}\right)\right)^{2} .
$$

4. Для доказательства логарифмического неравенства Соболева воспользуемся представлением (5) с $h=g^{2}, \mathrm{Eg}^{2}\left(B_{T}\right)=1$. Получим: $M_{u}=1+\int_{0}^{u} \mathrm{E}\left[h^{\prime}\left(B_{T}\right) \mid \mathscr{F}_{t}\right] d B_{t}$, где $h^{\prime}=\left(g^{2}\right)^{\prime}=2 g g^{\prime}$. Отсюда $d M_{t}=\mathrm{E}\left[h^{\prime}\left(B_{T}\right) \mid \mathscr{F}_{t}\right] d B_{t}, M_{0}=1$. Без ограничения общности можно считать, что $g^{2}\left(B_{T}\right)>0$ (п.н.). Тогда $d M_{t}=M_{t} d \widetilde{M}_{t}$, где $\widetilde{M}_{t}=$ $\int_{0}^{t} \mathrm{E}\left[h^{\prime}\left(B_{T}\right) \mid \mathscr{F}_{t}\right] / M_{t} d B_{t}, t \leqslant T$, есть локальный мартингал. Решение уравнения $d M_{t}=$ $M_{t} d \widetilde{M}_{t}$ с $M_{0}=1$ имеет вид $M_{t}=\exp \left\{\widetilde{M}_{t}-\frac{1}{2}\langle\widetilde{M}\rangle_{t}\right\}$, где $\langle\widetilde{M}\rangle$ есть квадратическая характеристика $\widetilde{M}$. Поэтому $M_{t} \log M_{t}=M_{t}\left(\widetilde{M}_{t}-\frac{1}{2}\langle\widetilde{M}\rangle_{t}\right)$ и по формуле Ито

$$
\begin{aligned}
d\left(M_{t} \log M_{t}\right) & =d\left(M_{t} \widetilde{M}_{t}\right)-\frac{1}{2} d\left(M_{t}\langle\widetilde{M}\rangle_{t}\right) \\
& =M_{t} d \widetilde{M}_{t}+\widetilde{M}_{t} d M_{t}+d\langle M, \widetilde{M}\rangle_{t}-\frac{1}{2}\langle\widetilde{M}\rangle_{t} d M_{t}-\frac{1}{2} M_{t} d\langle\widetilde{M}\rangle_{t} .
\end{aligned}
$$

Отсюда для моментов остановки $\tau_{n}=\inf \left\{t \leqslant T: M_{t} \geqslant n,\left|\widetilde{M}_{t}\right| \geqslant n\right\}$ находим, что $\mathrm{E}\left[M_{T \wedge \tau_{n}} \log M_{T \wedge \tau_{n}}\right]=\mathrm{E}\left\{\langle M, \widetilde{M}\rangle_{T \wedge \tau_{n}}-\frac{1}{2} \int_{0}^{T \wedge \tau_{n}} M_{t} d\langle\widetilde{M}\rangle_{t}\right\}=\frac{1}{2} \mathrm{E} \int_{0}^{T \wedge \tau_{n}} M_{t} d\langle\widetilde{M}\rangle_{t} \leqslant$ $\frac{1}{2} \mathrm{E} \int_{0}^{T} M_{t} d\langle\widetilde{M}\rangle_{t}$. Отсюда по лемме Фату и неравенству Коши-Буняковского

$$
\begin{aligned}
\mathrm{E} & {\left[M_{T} \log M_{T}\right] \leqslant \frac{1}{2} \mathrm{E} \int_{0}^{T} M_{t} d\langle\widetilde{M}\rangle_{t}=\frac{1}{2} \mathrm{E} \int_{0}^{T} \frac{\left(\mathrm{E}\left[h^{\prime}\left(B_{T}\right) \mid \mathscr{F}_{t}\right]\right)^{2}}{\mathrm{E}\left[h\left(B_{T}\right) \mid \mathscr{F}_{t}\right]} d t } \\
& =2 \mathrm{E} \int_{0}^{T} \mathrm{E}\left[\frac{g^{\prime}\left(B_{T}\right) g\left(B_{T}\right)}{\sqrt{\mathrm{E}\left[g^{2}\left(B_{T}\right) \mid \mathscr{F}_{t}\right]}} \mid \mathscr{F}_{t}\right] d t \leqslant 2 \mathrm{E} \int_{0}^{T} \mathrm{E}\left[\left(g^{\prime}\left(B_{T}\right)\right)^{2} \mid \mathscr{F}_{t}\right] \mathrm{E}\left[\frac{g^{2}\left(B_{T}\right)}{\mathrm{E}\left[g^{2}\left(B_{T}\right) \mid \mathscr{F}_{t}\right]} \mid \mathscr{F}_{t}\right] d t \\
& =2 \mathrm{E} \int_{0}^{T} \mathrm{E}\left[\left(g^{\prime}\left(B_{T}\right)\right)^{2} \mid \mathscr{F}_{t}\right] d t=2 T \mathrm{E}\left(g^{\prime}\left(B_{T}\right)\right)^{2} .
\end{aligned}
$$

Поскольку $M_{T}=g^{2}\left(B_{T}\right)$, то $\mathrm{E}\left[g^{2}\left(B_{T}\right) \log g^{2}\left(B_{T}\right)\right] \leqslant 2 T \mathrm{E}\left(g^{\prime}\left(B_{T}\right)\right)^{2}$, что при $T=1$ в предположении $\mathrm{E} g^{2}(\xi)=1$ дает логарифмическое неравенство Соболева (2).

\section{Список литературы}

[1] H. Chernoff, Ann. Probab., 9:3 (1981), 533-535. [2] L. H. Y. Chen, Z. Wahrscheinlichkeitstheor. verw. Geb., 69:2 (1985), 251-277. [3] Ch. A. J. Klaassen, Ann. Probab., 13:3 (1985), 966974. [4] В. В. Владимиров, Что такое математическая физика?, Препринт № HC-06/001, M., МИАН, 2006. [5] L. Gross, Amer. J. Math., 97:4 (1975), 1061-1083. [6] C. Ané, S. Blachère, D. Chafaï, P. Fougères, I. Gentil, F. Malrieu, C. Roberto, G. Scheffer, Panor. Synthèses, 10 (2000). [7] M. Ledoux, Lecture Notes in Math., 1709 (1999), 120-216. [8] I. Gentil, A. Guillin, L. Miclo, Probab. Theory Related Fields, 133:3 (2005), 409-436. [9] А. Ю. Шевляков, Теория вероятн. и матем. статист., 16 (1978), 159-163.

А. Н. Ширяев (А. N. Shiryaev)

Математический институт им. В. А. Стеклова РАН

E-mail: albertsh@mi.ras.ru
Представлено А. В. Булинским Принято редколлегией 02.05 .2006 